Адрес статьи / To link this article: http://cat.itmo.ru/ru/2021/v6-i1/266

\title{
Персонализация мобильного тура на основе стилей обучения
}

\author{
Е.В. Королёва \\ Университет ИТМО, Россия \\ zhenya.koroleva.2011@mail.ru
}

\begin{abstract}
Аннотация: Несмотря на разнообразие мобильных туров, многие из них однотипны и не предлагают никаких новаторских решений в сфере взаимодействия с пользователем. В данной работе будет описан инновационный метод персонализации, за основу которого взяты стили обучения типа ВАК (VAK). Идея состоит в предоставлении пользователю индивидуальной экскурсии, что учитывает его выбор репрезентации информации. Помимо этого, предполагается, что каждый тур продуман заранее, благодаря чему сохраняется связность повествования. Такая разновидность персонализации является многообещающей, так как стремится не только ответить на запросы туриста, но и заложить фундамент для дальнейших исследований в области опыта пользователя.
\end{abstract}

Ключевые слова: мобильный тур, персонализация, стили обучения, ВАК, экскурсия по городу, опыт пользователя.

С развитием технологий мобильные туры все больше становятся востребованными у туристов. Однако прогрессирует данная область достаточно медленно. Рынок наполнен однообразными приложениями, а инновационные проекты зачастую оказываются слишком дорогостоящими. В основном они связаны с внедрением дополненной реальности и геймификации, чем обусловлена трудность и затратность воплощения идей. Эти проекты представляют собой относительно новую траекторию развития мобильной экскурсии. Другой вектор развития, связанный с персонализацией и адаптацией экскурсии, тем временем оказался в фазе стагнации рис.1.

Каждый, у кого есть телефон, ощущает его продолжением себя. Поэтому важно, чтобы и само приложение, и его содержимое соответствовали индивидуальности пользователя. Таким образом, можно предоставить возможность выбирать не только тему экскурсии, но и способ репрезентации информации внутри нее. На этой идее строится новый подход, который должен учесть индивидуальные предпочтения каждого туриста на более глубоком уровне.

Более того, большинство мобильных туров нельзя назвать таковыми, если опираться на экскурсоводческую теорию. Многие из них могут называться лишь справочниками. Им не хватает основных признаков экскурсии: «единства цели, темы и задач экскурсии, и движения участников по заранее запланированному маршруту для осмотра объектов с использованием экскурсионного метода» $[1$, c.56]. Эти особенности были взяты из учебника по экскурсоведению Н.А. Добриной [1], который является основным пособием для экскурсоводов. Мобильные гиды, прежде чем стать 
действительно тем, чем они называются, должны значительно улучшиться. В связи с этим целью работы является предложение нового способа персонализации и методики по преобразованию тура. В результате решатся две вышеозвученные проблемы: нехватка значительных разработок в сфере персонификации и отсутствие фактической экскурсии в мобильном приложении.
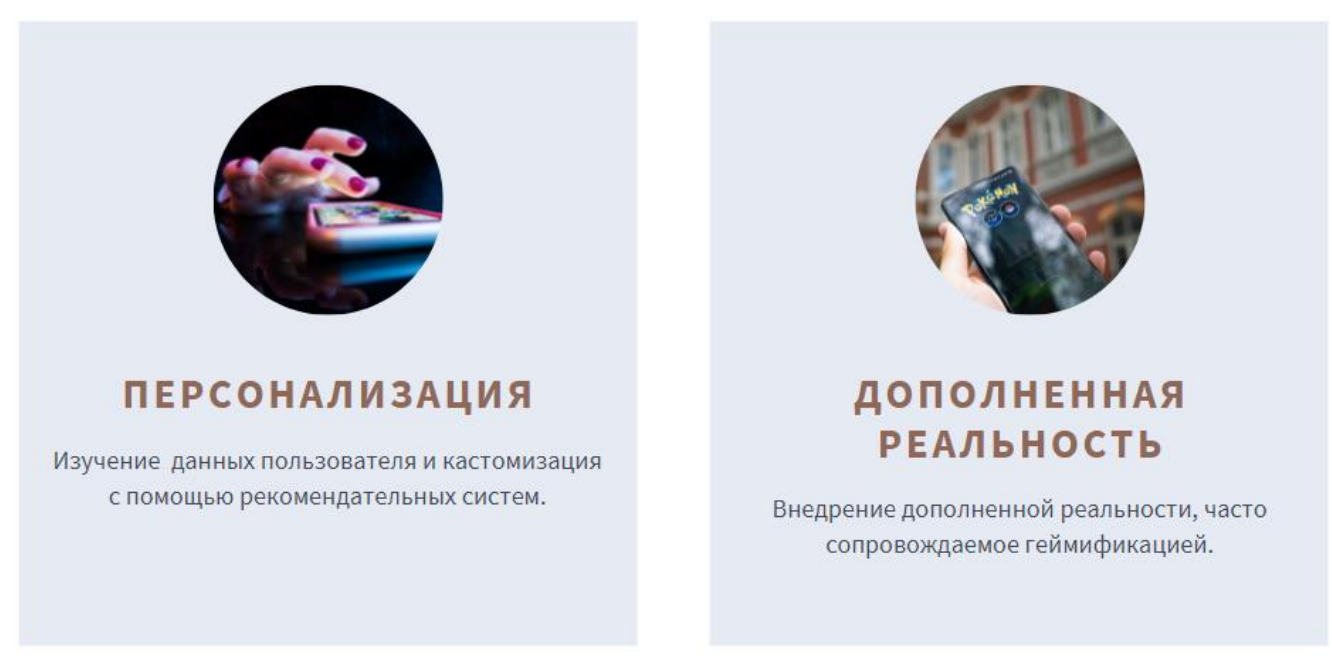

Рис. 1. Две траектории развития экскурсии

За основу персонализации, где учитывается тип репрезентации информации в соответствии с запросами каждого пользователя, были взяты исследования в сфере стилей обучения. Их активный период пришелся на 80-90-е годы XX века. Статьи были направлены на то, чтобы определить, как разные люди предпочитают получать информацию во время обучения и классифицировать эти предпочтения. Категории или типы стилей обучения могут варьироваться от работы к работе, потому как в научном контексте нет установившегося определения того, что такое стили обучения. Их рассматривают с разных точек зрения, всегда добавляя некоторые новые детали в дефиницию. В этой работе под стилями обучения понимается предпочтение в способе получения информации во время учебы. Данная трактовка близка к психологической точке зрения на термин. Это можно объяснить тем, что новый способ персонализации, предлагаемый в работе, основан на теории стилей обучения Н.Д. Флеминга VARK [2], которая касается области психологии.

Она была описана в статье «Я другой; не тупой. Способы презентации (В.А.Р.К.) на занятиях в высших учебных заведениях» [2]. Теория характеризуется сенсорным подходом к идентификации стилей обучения. Она основана на НЛП [нейролингвистическом программировании] и некоторых теориях об исследованиях левого и правого полушарий. Однако автор подчеркивает, что «то, что студенты получают [от этой работы], - это не НЛП или ускоренное обучение, или упражнения для левого /правого полушария мозга, а набор стратегий восприятия информации, соответствующих их сенсорным предпочтениям» [2, с. 309].

Н.Д. Флеминг выделяет четыре стиля обучения, которые соответствуют аббревиатуре VARK. «V» означает способ получения информации в визуальной форме: изображения, видео, графики, карты и т. д. «А» относится к аудиальному предпочтению: подкасты, аудио рассказы, лекции, музыка и прочее. Студенты склонны «учиться на слух». Следующая буква «R» - это аббревиатура стиля обучения «Read and Write» (читать и писать), который включает в себя предпочтение в классических задачах чтения и письма. Другими словами, это означает восприятие информации через текст. Последняя буква «К» соответствует кинестетическому способу получения информации. Он подразумевает тяготение к обучению через опыт, требующий использования всех органов чувств. Людей, предпочитающих действие, движение, в данном случае называют кинестетиками. «Они изучают теорию через ее применение» [2, с.309].

Автор видит новизну в том, чтобы добавить четвертое модальное деление (чтение и письмо) к более ранней концепции НЛП, указав визуальное измерение. Это выделение может быть 
полезным при работе и применении теории в классе, но оно идет вразрез с реалиями мобильного гида. Во время прогулок у пользователей не так много времени, как и желания, для письма или продолжительного чтения. Вместе с тем более детальное различение внутри визуального канала может вызвать путаницу на начальных этапах работы с приложением. Целесообразнее не указывать измерение «чтение и письмо», поскольку оно будет в любом случае удовлетворено посредством текста в категории визуального. Вдобавок, мало кто из исследователей придал данному разделению большое значение.

Решение предпочесть теорию Н.Д. Флеминга другим обусловлено тремя фактами. Она наиболее популярна и широко используется. Более того, эта концепция кажется интуитивно понятной многим людям вне академического сообщества. Итак, представляется возможным эффективно применить ее в контексте экскурсии мобильного гида. В этом исследовании есть намерение использовать стили обучения лишь как способ углубить персонализацию. Их эффективность в учебном процессе - спорная тема, которая не будет упоминаться ввиду нерелевантности. Для этой работы не имеет значения, приводят ли стили обучения к лучшему запоминанию информации или нет. Мобильный гид - это не только источник увлекательной информации, но и способ насладиться городом, получить незабываемые впечатления. Соответственно, удовлетворенность пользователя играет решающую роль. Люди ценят персонализацию, и подход, применяющий стили обучения, может повысить заинтересованность приложением. У пользователей появляется дополнительная возможность показать свою индивидуальность и обозначить интересы путем выбора наиболее подходящего способа представления информации.

При оценке чего-либо на первый план все больше выходит эмоциональная реакция. Турист будет воспринимать контент с позиции «нравится / не нравится». В наши дни это общемировая тенденция. Достаточно вспомнить соответствующие кнопки во всеобъемлющих социальных сетях. Для пользователя не важна корреляция между способностями к обучению и способом представления, пока он доволен тем, что получает от приложения. Средняя продолжительность внимания уменьшается, особенно среди поколений $\mathrm{Y}, \mathrm{Z}$ и А, которые будут потенциальными клиентами данного мобильного приложения. В связи с этим необходимо предоставить информацию в туре интересным способом и дать туристу самому решать, как он хочет его проходить: слушая ли только аудио, смотря ли картинки и читая текст или же играя и решая головоломки.

Table 1

\begin{tabular}{lll}
\hline Auditory & Visual & Kinesthetic \\
\hline Discussion & Texts & Movement \\
Debate & Charts & Role-plays \\
Podcasts & Tables & Drama \\
Dictations & Graphs & Races and competitions \\
Jigsaw reading & Mind maps & Handling objects or props \\
Reading aloud & Graphic Organizers & \\
Storytelling & Art & \\
Chain games/chants & Drawings & \\
Lectures & Pictures & \\
& Posters & \\
& Realia & \\
& Visualizations & \\
\hline
\end{tabular}

Рис. 2. Список заданий для каждого типа репрезентативной системы 
В приложении, что будет использовать метод персонализации, основанный на типах репрезентативных систем, каждая локация должна иметь три варианта представления пользователю по системе ВАК (VAK). То, какие задания и материалы соответствуют каждому типу репрезентации, было указано в статье Лизы Леопольд [3] в таблице на рис. 2. Однако экскурсия по городу имеет другой контекст, поэтому многие из них должны быть адаптированы. Также не удается избежать полного отказа от текстовой информации. Когда говорится о том, что сейчас у определенной локации выбран тип репрезентации «аудио», это значит, что соответствующие ему материалы преобладают. Задания на движение в большинстве своем сводятся к взаимодействию с телефоном, играм и решениям интересных задач на локации. Стоит отметить, что пользователю не следует сразу попадать на страницу с материалами. Вначале ему должна быть представлена картинка достопримечательности, по которой он дополнительно сможет сориентироваться и точно подойти к нужному месту на карте тура.

То, какой вариант репрезентации представлен у локации, зависит от выбора, что был сделан до того, как начать тур. Процесс должен выглядеть следующим образом. На экране отобразятся 3 иконки со слайдерами. Каждая из них отразит один тип репрезентации информации. Пользователь должен установить их процентное содержание в экскурсии и посмотреть справку, если возникают трудности. Так, например, он может выбрать $100 \%$ аудиальный тип, тогда получит аудиогид. А может выбрать разные соотношения типов, как на рис. 3. В любом случае система будет подстраиваться под его предпочтения и выдавать материал, соответствующий данной пропорции. Таким образом, из 6 локаций в туре описание трех может оказаться с преобладанием аудиофайлов, двух с заданиями и играми, а одной преимущественно с картинками и видео. Каждый турист в результате получит индивидуальный тур. Он обязательно должен быть заранее продуман, и автоматически в нем будет происходить лишь пропорциональное соотношение типов репрезентации. Это значит, что данная экскурсия претендует на обладание всеми ее основными признаками с теоретической точки зрения.

\section{ВЫБОР ПользовАтЕлЯ}
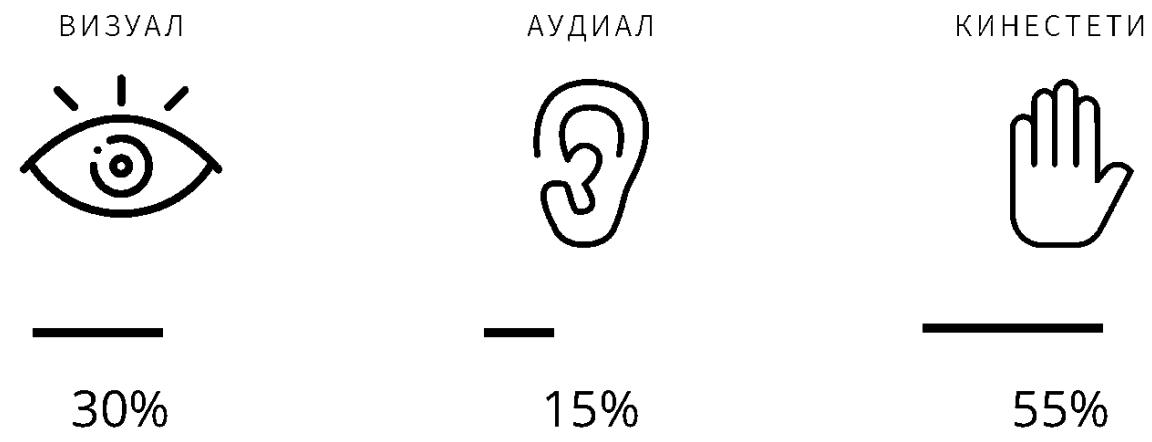

Рис. 3. Процесс выбора репрезентации информации

Несомненно, такой подход к персонализации обладает рядом преимуществ, который обоснован исследованиями в области опыта пользователя (англ. user experience). Один из главных ученых в данной сфере, Марк Хассенцаль, говорит о двух аспектах восприятия интерактивного продукта: прагматическом и гедонистическом. Он объясняет, что прагматическое измерение соответствует полезности и удобству использования продукта, а гедонистическое измерение означает «более общие человеческие потребности, выходящие за рамки инструментальных такие, как потребность в новизне и изменениях, личностном росте, самовыражении и / или связанности» [4, с. 12]. Большинство этих потребностей будет удовлетворено посредством использования нового метода персонализации. Предполагается также продолжительный положительный эффект на пользователя. Без желания преуменьшить важность прагматической составляющей приложения можно отметить, что с большей вероятностью турист вспомнит, как

International Culture \& Technology Studies, Vol. 6, No. 1 
относился к мобильному туру, а не его стабильную работу. Поскольку люди не пользуются такого типа приложениями ежедневно, важно мотивировать их вернуться к нему, когда оно им понадобится. Следственно, этот инновационный подход к персонализации, помимо всего прочего, призван обеспечить долгую жизнь приложения на рынке.

Несмотря на все преимущества, есть и ряд недостатков. Приложение предполагает задействование большого количества человеческих ресурсов. Каждая экскурсия должна быть продумана со стороны трех разных типов восприятия. Поиск и создание соответствующих материалов также потребует времени. Помимо этого, не всегда к локации легко подобрать нужное задание. Их вариативность ограничена. Что касается пользователя, то предоставление экскурсии в онлайн-формате все еще предполагает риск технических неполадок, связанных с нестабильным Интернет-соединением.

В заключение этот новый метод персонализации пользователя основан на внедрении теории стилей обучения, основанных на восприятии. Такой подход поможет не только обратить внимание на предпочтения пользователей, но и обеспечить новый опыт работы с профессионально созданными маршрутами. Пользователи приложения будут выбирать процент информации определенного типа, которую они хотят видеть в туре, поэтому частично получат ответственность за свой опыт прохождения маршрута. Для дальнейших исследований можно собрать данные об их выборе. Это было бы довольно легко осуществимо, поскольку «потребители готовы предоставить значительную личную информацию в обмен на признание и лучшие услуги» [5, с. 613]. Таким образом, появится возможность создать более сложную систему рекомендаций, проанализировать поведение пользователя во время прохождения тура, отследить предпочтения большинства в типах репрезентации и задаться новыми научными вопросами.

\title{
Литература
}

[1] Добрина Н. А. Экскурсоведение: учебное пособие. М.: Флинта: МПСИ, 2012. 285 с.

[2] Fleming N. D. I'm different; not dumb. Modes of presentation (VARK) in the tertiary classroom //Research and development in higher education, Proceedings of the 1995 Annual Conference of the Higher Education and Research Development Society of Australasia (HERDSA), HERDSA.1995. V. 18. P. 308-313.

[3] Leopold L. Prewriting tasks for auditory, visual, and kinesthetic learners //TESL Canada Journal. 2012, №. 29. P. 96-102.

[4] Hassenzahl M. User experience (UX) towards an experiential perspective on product quality //Proceedings of the 20th Conference on l'Interaction Homme-Machine. 2008. P. 11-15.

[5] Buhalis D., Law R. Progress in information technology and tourism management: 20 years on and 10 years after the Internet-The state of eTourism research //Tourism management. - 2008. V. 29, №. 4. P. 609-623.

\section{Personalization of Mobile Tour Guides Based on Learning Styles Approach}

\author{
E.V. Koroleva
}

ITMO University, Russia

\begin{abstract}
Modern so-called mobile guided tours are just advanced electronic guidebooks. Theoretically speaking, it is impossible to call them a guided tour because they lack fundamental features: the unity of purpose, theme, and objectives of the guided tour; and movement of tour participants along a pre-planned route to view objects using the sightseeing method. Moreover, the scientists mainly devote their studies to the implementation of technical features and recommendation algorithms, while the system lacks various information representation and deep interaction with the user.

This research offers a new method to personalize and transform the tour. It takes learning styles approach described by Fleming. His theory is built on NLP [Neuro-Linguistic Programming] and some theories about left brain and right brain studies. There are three main preferences of the information perception corresponding to the following abbreviation VAK (Visual, Audial, Kinesthetic). The idea is that users of the app will choose the percentage of the information of a certain type they want to see. Later he will be provided with the individual tour according to his preferences in representation. In other words, the system will adjust to the given ratio and return the
\end{abstract}


material corresponding to this proportion. Thus, out of 6 locations in the tour, the description of three may be dominated by audio files (A), two by tasks and games (K), and one mainly filled with pictures and videos $(\mathrm{V})$. Each tour will be constructed in advance with the information adaptation. Therefore, the tour users will get is coherent and meet the standards of the theory of tour creation. In addition, the interaction with the software will be more meaningful and presumably may provide stronger emotional attachment over time because the application will address individual requirements. For further studies, it is possible to collect information about users' choices to create a more complex system of recommendations.

Keywords: mobile tour guide, personalization, learning studies, VAK, city tour guide, user experience.

\section{References}

[1] Dobrina N.A.(2012). Jekskursovedenie: uchebnoe posobie [Tour guide studies: text book]. Moscow. Flinta: MPSI. $285 \mathrm{p}$.

[2] Fleming N. D.(1995). I'm different; not dumb. Modes of presentation (VARK) in the tertiary classroom. Research and development in higher education, Proceedings of the 1995 Annual Conference of the Higher Education and Research Development Society of Australasia (HERDSA), HERDSA. Vol. 18. 308-313.

[3] Leopold L.(2012) Prewriting tasks for auditory, visual, and kinesthetic learners. TESL Canada Journal. No.29. 96-102.

[4] Hassenzahl M.(2008) User experience (UX) towards an experiential perspective on product quality. Proceedings of the 20th Conference on l'Interaction Homme-Machine. 11-15.

[5] Buhalis D., Law R.(2008) Progress in information technology and tourism management: 20 years on and 10 years after the Internet—The state of eTourism research. Tourism management. Vol. 29, No.4. 609-623. 